\title{
Rate of intracranial self-stimulation as a function of reinforcement magnitude and density
}

\author{
Bernard Beer, William Hodos and T. J. Matthews \\ WALTER REED ARMY INSTITUTE OF RESEARCH
}

\begin{abstract}
Rate-intensity functions for rewarding intracranial stimulation (ICS) were compared for a continuous reinforcement schedule (CRF) and several variable interval schedules (VI). The rate of CRF responding declined at the higher intensities of ICS. With relatively longer VI schedules, however, self-stimulation rate increased as a monotonic function of intensity.

\section{Problem}

The rate of intracranial self-stimulation (ICS) has been frequently used as the index of reward strength. The relationship between the intensity of ICS and the rate of self-stimulation in many areas of the brain has been shown to reach a maximum at intermediate intensities and then to decline at higher intensities (Reynolds, 1958). This decline in self-stimulation rate at high intensities has been attributed to the spread of current from the positive reward cells in the vicinity of the electrode tip to nearby areas yielding punishment effects (Olds \& Olds, 1963). On the other hand, preference tests (Hodos \& Valenstein, 1962) have indicated that rats will consistently choose the more intense of two stimuli even though they then self-stimulate at a lower rate. The present experiment was designed to analyze this paradoxical finding in terms of the temporal properties of the reinforcement schedule.
\end{abstract}

\section{Method}

The subjects were three male albino rats from the colony of the Walter Reed Army Institute of Research. During the course of the experiment, the animals were permitted free access to food and water in the home cage. ${ }^{1}$ Each rat was stereotaxically implanted with a bipolar, stainless steel electrode which was bare only at the cross-sectioned tips. At the termination of the study the rats were sacrificed, perfused with saline and formalin and their brains were sectioned and stained with cresylecht violet. The locations of the electrode tips were: $\mathrm{PH}-24$, lateral tegmental nucleus; $\mathrm{PH}-26$, lateral hypothalamus; PH-28, ventral tegmental nucleus.

The current of the ICS ranged from 0.1 to $4.0 \mathrm{ma}$, zero to peak. The wave form and other parameters of the ICS have been described elsewhere (Hodos \& Valenstein, 1962). The experimental conditions were programmed and the data collected by a system of relay switching circuits, timers, counters, and cumulative recorders.

Following initial lever-pressing training, a rateintensity function was obtained for each rat. The stimulation was delivered on a continuous reinforcement (CRF) schedule in which each lever press was reinforced with stimulation. Three intensities were then

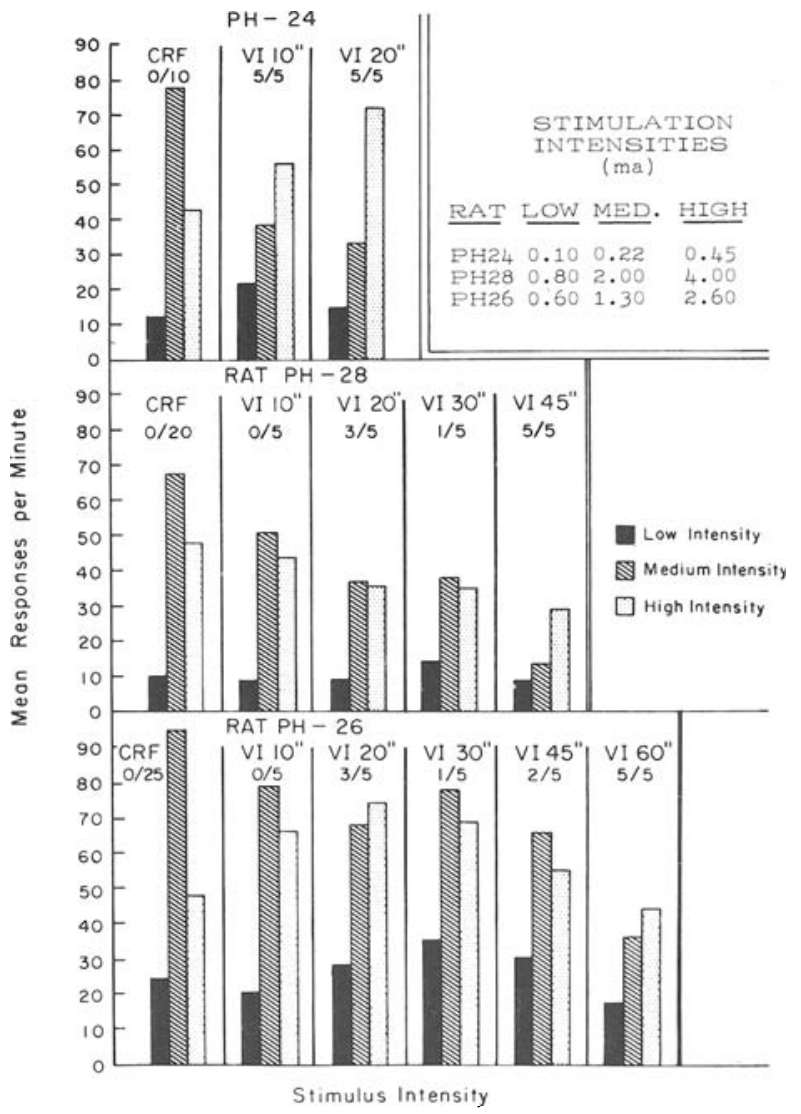

Fig. 1. Rate of self-stimulation has been plotted as a function of stimulus intensity for each rat. Data are shown for CRF and each of the VI schedules used. Stimulation intensities for each rat are shown in the upper right of the figure.

chosen for each rat. Low intensity was slightly above the threshold for the maintenance of self-stimulation behavior. Medium intensity was at the peak of the rateintensity function. High intensity was twice the current of medium intensity and invariably resulted in a marked depression of the self-stimulation rate. The intensities for each rat are shown in the upper right portion of Fig. 1.

The animals were then trained on a variable interval (VI) schedule in which lever responses were intermittently reinforced with ICS of medium intensity after a variable period of time had elapsed since the previous reinforcement. The mean time between reinforcements was $10 \mathrm{sec}$. (VI 10). When performance had stabilized on 
the VI schedule, a new rate-intensity function was obtained using the three previously selected intensities. The intensities were presented in an irregular sequence of $5 \mathrm{~min}$. test periods until a total of three tests at each intensity were obtained. Rate-intensity functions were obtained using the CRF and VI 10 schedules on alternate days for a total of 10 days. The procedure was then repeated with progressive increases in the average interval of the VI schedule until the rate-intensity function obtained from each rat on all five VI days was monotonic; i. e., the highest self-stimulation rate was observed at the highest ICS intensity and the lowest rate at the lowest intensity.

\section{Results}

The results are shown in Fig. 1. The numbers above each set of bar graphs indicate the number of days on which the rate-intensity function was monotonic. Thus $3 / 5$, for example, would indicate monotonicity on 3 out of 5 test days. The CRF data which were collected alternately with the VI data have been combined into a single set of bar graphs since they showed little variation from one condition of the experiment to the next. The results indicate that for each rat, as the length of the VI was increased, a monotonic rate-intensity function was eventually obtained. With rat PH-24, VI 10 was sufficient to produce monotonicity on 5 out of 5 VI days. Rat PH-28 did not show monotonicity until the schedule had been adjusted to VI 45. Rat PH-26 yielded a monotonic function on all 5 test days only when the average interval of the VI schedule had been increased to $60 \mathrm{sec}$.

\section{Discussion}

Two recent studies support the data reported here. In the first of these, Steiner (1964) obtained monotonic rate-intensity functions using a fixed ratio schedule. While his experiment supports the finding that monotonic functions can be obtained with intermittent reinforcement, he did not explicitly manipulate the temporal distribution of reinforcement as was done in the present experiment. In the second study, by Hawkins \& Pliskoff (1964), rate-intensity functions were obtained using a two-member behavioral chain. The first member of the chain was a VI 30 schedule and the second member was a CRF. The reinforcement for lever-pressing on the
VI member of the chain was access to the CRF member. Their data indicate that the rate of responding in the CRF member of the chain declined at higher intensities whereas the rate in the VI 30 member increased with increasing intensities of stimulation.

An alternative to the notion of spread of current into negative areas as an explanation of the decline of selfstimulation rate at high intensities is that current spreads into areas which are principally motor. The resultant competing behavior (tremors, seizures and forced movements) interferes with the smooth execution of the lever-pressing response and the rate of responding declines. Intermittent schedules minimize the effect of these competing responses by reducing the density of reinforcement. Moreover, as stimulations are further dispersed in time by increasing the length of the VI, the influence of the motor side effects on the rate-intensity function becomes negligible. Thus, by minimizing the temporal density of reinforcement, we have been able to reconcile the rate-intensity function with the data obtained from intensity preferences.

\section{References}

HAWKINS, T. D., \& PLISKOFF, S. S. Brain-stimulation intensity, rate of self stimulation, and reinforcement strength: An analysis through chaining. J. exp. Anal. Beh.', 1964, 4, 285-288.

HODOS, W., \& VALENSTEIN, E. S. An evaluation of response rate as a measure of rewarding intracranial stimulation. J. comp. physiol. Psychol., 1962, 55, 80-84.

OLDS, M. E., \& OLDS, J. Approach-avoidance analysis of rat diencephalon. J. comp. Neurol., 1963, 120, 259-295.

REYNOLDS, R. W. The relationship between stimulation voltage and rate of hypothalamic self-stimulation in the rat. J.comp. physiol. Psychol., 1958, 51, 193-198. STEINER, S. S. Effects of current intensity and schedule of reinforcement on response rate for intracranial electrical stimulation in the rat. Eastern Psychological Association, 1964, Philadelphia.

\section{Note}

1. The principles of animal laboratory care as promulgated by the National Society for Medical Research were observed. 